\title{
ETCHING TECHNIQUE TO STUDY PLASTIC DEFORMATION OF ICE
}

\author{
By A. S. Krausz \\ (Division of Building Research, National Research Council, Ottawa, Canada)
}

\begin{abstract}
When ice is kept in saturated air, thermal etching occurs. This has been applied in the study of grain boundary migration, sub-boundary and slip line formation. A few of the interesting results
are reported to illustrate the usefulness of the method.

RÉsumé. Lorsque de la glace est maintenue dans une enceinte d'air saturée, elle est soumise à la corrosion thermique par sublimation. Cette technique a été utilisée pour étudier la migration des surfaces de séparation entre les grains, la formation de nouvelles parois et de lignes de glissement. Quelques uns des résultats intéressants sont mentionnés pour illustrer l'utilité de la méthode.
\end{abstract}

Zusammenfassung. Hält man Eis in gesättigter Luft, so unterliegt es der thermischen Ätzung. Diese Tatsache wurde zum Studium der Veränderung der Korngrenzen sowie der Bildung von Feinkorngrenzen und Gleitebenen herangezogen. Einige der interessanten Ergebnisse werden mitgeteilt, um die Zweckmässigkeit dieser Methode zu zeigen.

In the study of the creep behaviour of ice, much work has been done with the aim of establishing the stress-strain and strain-time relationships but comparatively little has been done to establish the underlying and governing physical phenomena. Current investigations at the Division of Building Research, National Research Council of Canada are directed toward this neglected aspect of investigations on ice. In particular, attention is being paid to the development of methods for observing features at the ice surface during the deformation process. It is the aim of this note to report one technique developed and to draw attention to some of the interesting observations obtained with it.

According to the present view, plastic deformation is closely connected with irregularities present in the crystals. In their immediate neighbourhood, these irregularities (dislocations) disturb the energy state of the crystal and thus affect the chemical, mechanical, and some of the other physical properties at this location. The rate of evaporation of ice depends on the free energy difference between the evaporating surface and the water vapour in the medium surrounding that surface. It is possible, therefore, to make visible the disturbed sites with their higher free energy by choosing the correct conditions of evaporation. It has been found that when ice evaporates very slowly, the disturbed regions soon become visible because of the faster evaporation at these sites. This is one of the effects on which the technique of thermal etching is based.

To obtain the best result it is necessary to polish the specimen toa mirror-like finish before thermal etching. This is done by rapid evaporation for a sufficient length of time in a very dry atmosphere. Under the conditions used for the observations described in this note, this time was usually less than $30 \mathrm{~min}$. Placing the specimen in an air-tight box in the presence of sodium hydroxide or calcium chloride proved to be a practical method of controlling the progress of the surface polish. For the thermal etching proper, the specimen is placed in an air-tight box partially filled with small pieces of ice to provide a saturated atmosphere. For the present observations, the specimens were beams to which was applied a four-point load with a special apparatus completely contained within the box. Microscopic observations of the ice surface were made through the transparent top of the box during the deformation.

Grain boundaries show up distinctly within a few minutes using this technique (see Fig. I, p. I005). As the creep progresses, grain boundary migration is sometimes observed; the old boundary slowly disappears and the new boundary appears as a well-defined line. An example of this is shown in the right-hand side of Figure 2. Another interesting example is shown in Figure 3 where the grain boundary migration proceeded to such an extent that the small middle crystal was consumed by the surrounding four larger crystals. Sometimes one or more previous 
locations of the migrated boundary show up simultaneously, indicating that the migration of the grain boundary occurs in jumps (see Figs. 2, 3, and 4).

It was often observed that two, mutually perpendicular, families of thin lines developed during creep or recovery as illustrated in Figure 4 . The grain boundaries which intersect the orthogonal line features are usually very jagged. An example is shown in Figure 4. There are indications that one family of the lines is in the basal plane and the other parallel to the $c$-axis. Therefore it is anticipated that they are connected with slip lines and polygonized boundaries respectively.

Additional observations on the creep process have been carried out; further reports are being prepared.

This is a contribution from the Division of Building Research, National Research Council, Canada, and is published with the approval of the Director of the Division. 
Fig. I. Grain boundaries, etched before deformation $(\times 25)$
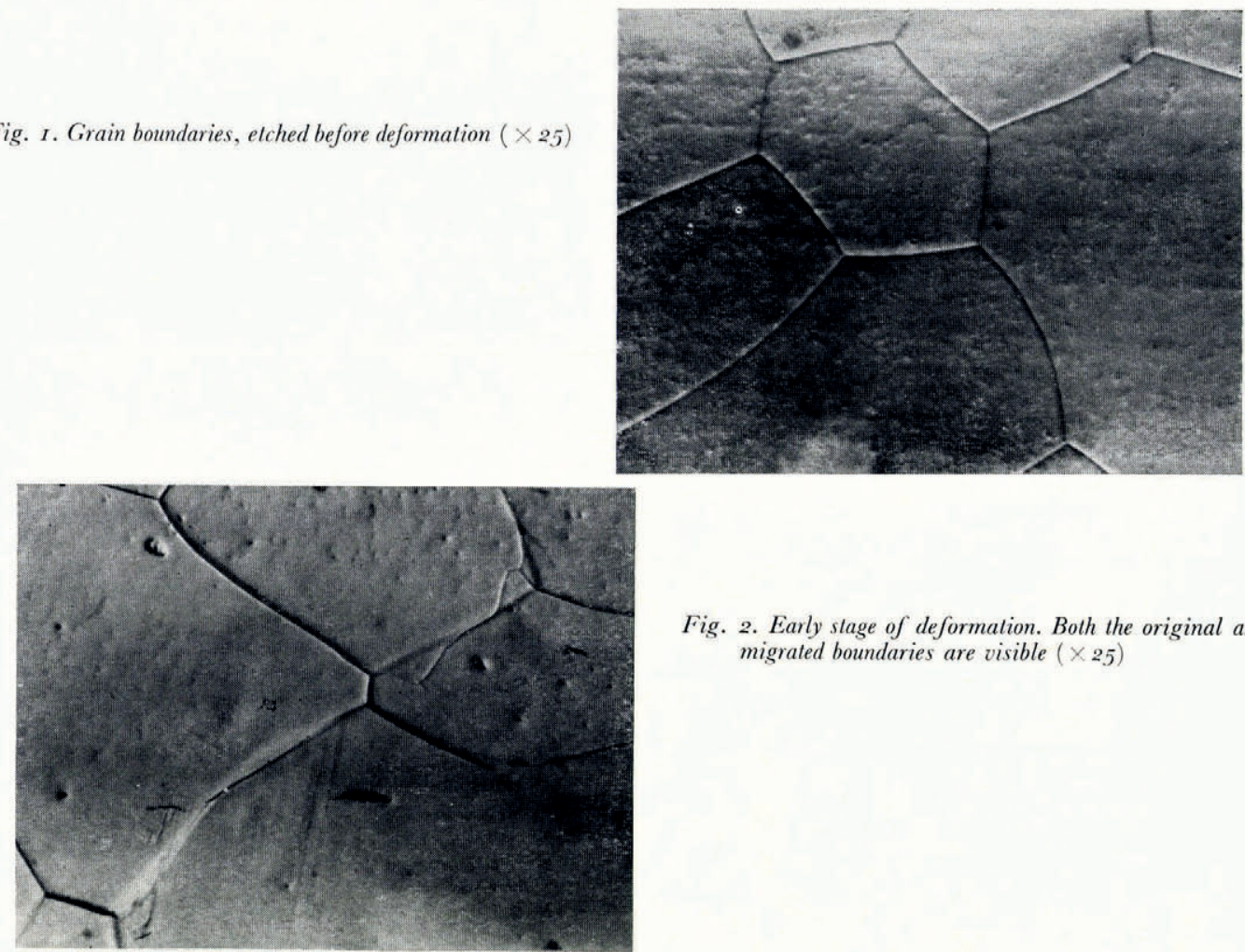

Fig. 2. Early stage of deformation. Both the original and migrated boundaries are visible $(\times 25)$

Fig. 3. An example of a small grain consumed by the surrounding big crystals $(\times 25)$
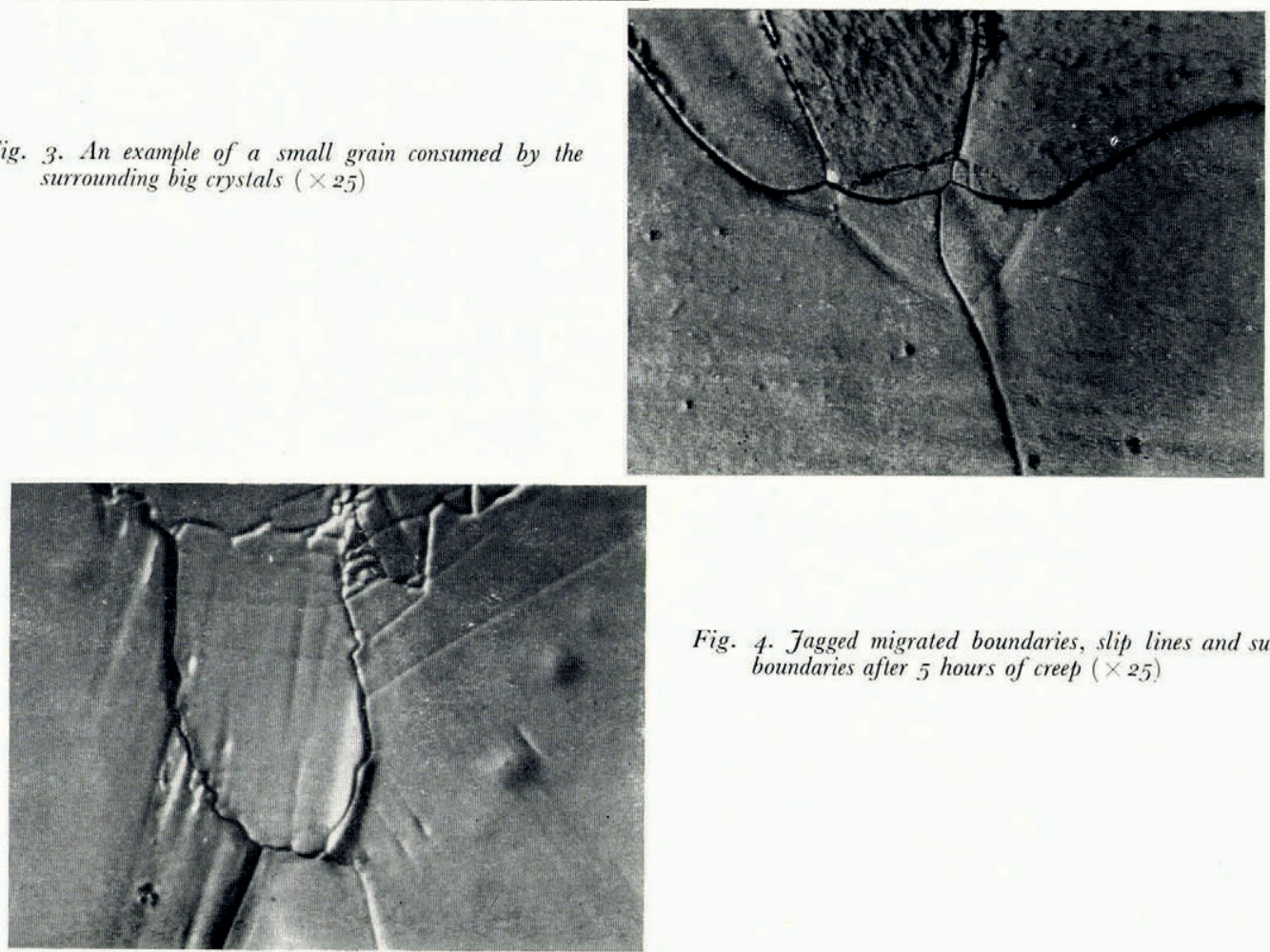

Fig. 4. Jagged migrated boundaries, slip lines and subboundaries after 5 hours of creep $(\times 25)$ 